\title{
Le métathéâtre cubain et la comédie du pouvoir : du spectacle de l'échec à celui de l'espoir?
}

\section{Séverine Reyrolle}

\section{(2) OpenEdition}

1 Journals

Édition électronique

URL : https://journals.openedition.org/cher/12454

DOI : $10.4000 /$ cher.12454

ISSN : 2803-5992

Éditeur

Presses universitaires de Strasbourg

\section{Édition imprimée}

Date de publication : 30 juin 2012

Pagination : 213-223

ISBN : 978-2-35410-046-9

ISSN : 1968-035X

\section{Référence électronique}

Séverine Reyrolle, "Le métathéâtre cubain et la comédie du pouvoir : du spectacle de l'échec à celui de l'espoir? », reCHERches [En ligne], 8 | 2012, mis en ligne le 21 février 2022, consulté le 23 février 2022. URL : http://journals.openedition.org/cher/12454 ; DOI : https://doi.org/10.4000/cher.12454

\section{cc) (†) (2)}

Ce(tte) œuvre est mise à disposition selon les termes de la Licence Creative Commons Attribution Pas d'Utilisation Commerciale - Partage dans les Mêmes Conditions 4.0 International. 


\title{
Le métathéâtre cubain et la comédie du pouvoir: du spectacle de l'échec à celui de l'espoir?
}

\author{
Séverine Reyrolle \\ Université Paris Ouest Nanterre La Défense
}

$\ll \int \begin{aligned} & \text { et'aime... moi non plus... », tel pourrait-être le refrain des tumultueuses } \\ & \text { et antiques liaisons entre théâtre et politique. Ainsi, lorsque le peuple }\end{aligned}$ cubain est confronté à la désillusion révolutionnaire et l'oppression d'un nouveau régime totalitaire, lorsque ses artistes affrontent soudain une censure massive entravant jusqu'aux racines de leur liberté créatrice, plus que jamais, cette petite musique subversive ressurgit et érige alors le métathéâtre en une technique de contestation dramaturgique privilégiée. Chez José Triana et Matías Montes Huidobro, le théâtre dans le théâtre devient en effet l'instrument idéal pour déjouer les interdits et dénoncer «en catimini » cette comédie politique que sont devenus le gouvernement et la société castriste! Autrement dit, ces vertigineux enchâssements multiples leur permettent d'exprimer non seulement ces tragiques bouffonneries que sont les affaires publiques aujourd'hui, mais aussi cette farce grotesque à laquelle se résume désormais leur propre vie. Du macrocosme sociétal au microcosme familial, du theatrum mundi au theatrum intimi, le métathéâtre cubain révèle en somme l'étendue du désenchantement qui envahit ces hommes violemment. Reste à savoir si le théâtre dans le théâtre cubain en reste à ce triste constat d'aliénation ou si, au contraire, il témoigne d'une volonté nouvelle d'adaptation et d'émancipation. Au cours de notre étude, nous nous attacherons donc à montrer que le métathéâtre cubain dessine peut-être moins une psychopathologie de l'échec et de la frustration qu'une tentative de réconciliation et de reconstruction... Contre toute attente, en 
effet, il arrive parfois que, de la comédie du pouvoir et du spectacle dérisoire d'une humanité névrosée, surgisse in fine une lueur inébranlable d'espoir résidant dans une créativité renouvelée.

Sous la plume des dramaturges cubains, le théâtre dans le théâtre apparaît tout d'abord comme une métaphore parfaite de cette vaste mascarade politique qu'est la révolution castriste. Dès la Noche de los asesinos, en effet, José Triana met en place une structure dramaturgique spéculaire qui mime et interroge subversivement la valeur réelle de la révolution cubaine. De fait, les trois frères et sœurs de sa pièce répètent une cérémonie qui n'a d'autre sujet, elle aussi, qu'une profonde volonté de changement et une révolte face à une autorité paternelle arbitraire. Or, très vite, on constate qu'ils ne font que reproduire à tour de rôle le schéma du pouvoir oppresseur et violent qui les a pourtant contraints à s'enfermer dans ce jeu intérieur devenu perpétuelle conspiration. Ainsi, d'emblée, le métathéâtre circulaire de Triana remet clairement en cause le sens profond et la réalité même de la révolution. Ceci est plus frappant encore dans une création postérieure de l'auteur, intitulée Ceremonial de guerra. Dès le titre en effet, Triana approfondit l'analogie «déréalisante» entre théâtre et politique en révélant ostensiblement le caractère « cérémonial », c'est-à-dire artificiel, des stratégies et des prétentions guerrières révolutionnaires. Puis, tout au long de la pièce, ses personnages militaires apparaissent comme des acteurs étonnamment conscients de leur statut dramatique au sein de cette vaste comédie politique qu'est, pour eux aussi, l'assaut d'un minuscule fort symbolisant le contrôle du territoire insulaire. "Supongo que jugamos un papel ¿no?» (Triana 1990: 19) s'écrie en effet le soldat Juan, dès la scène 2, juste avant que Felipe ne s'exclame triomphalement: «¡Aquí nos la jugamos toda!»(30). Ainsi, peu à peu, ces jeux de rôles métathéâtraux brouillent également les frontières entre fiction et réalité et parviennent à montrer que «la vérité révolutionnaire» - cette «vérité» qui sera répétée pas moins de cinquante fois dans la pièce n'est aussi finalement qu'une chimère mensongère, qui restera à jamais, à l'image de la carte du fort, introuvable. «Tu verdad y mi mentira... Sueños sueños...» (58) conclut en effet brillamment Carlos... Enfin avec Fiesta, en 1992, la dénonciation de la farce politique castriste de Triana atteint son paroxysme puisque pour la première fois la comédie intérieure associe clairement Fidel Castro à un metteur en scène et un imposteur émérite. En effet, devant les exigences et les règles quasi dictatoriales que Gerardo impose à sa famille pour jouer sa comédie festive, Don Benito demande «¿Es un asunto de poder, o una fiesta? [...] ¡es que tú te has inventado una 
nueva democracia como el hombre de las barbas!...» (Triana 1992: 272). Et Montes Huidobro de parachever cet habile procédé en évoquant, dans Exilio, les discours de Fidel, puis en faisant clamer à ses personnages, immédiatement après: « todo lo que ha pasado aquí en los últimos años haya sido una farsa. [...] Puro teatro. Teatro nada más. Teatro nacional. Eso sí, un teatro de "Patria o muerte" que nos puede costar la vida" (Montes Huidobro 1988: 43). Ainsi, que ce soit chez Triana ou chez Huidobro, le métathéâtre finit toujours par montrer que la révolution cubaine est le véritable et unique théâtre de l'île.

Gare aux apparences ou aux conclusions hâtives cependant! Car le métathéâtre cubain ne s'en tient pas à cette habile démonstration de la spectacularisation du macrocosme politique. Il révèle également l'artificialité du microcosme sociétal dans son intégralité. En effet, les pièces intérieures de Triana et de Montes Huidobro mettent ensuite au jour la comédie sociale des relations familiales et amicales. Dans Funeral en Teruel par exemple, l'actrice Isabel s'enferme dans la récitation de pièces romantiques puis dans la représentation d'une mort imaginaire car elles seules lui permettent de conserver l'illusion d'une union amoureuse sincère et choisie. La fin de son intra-jeu et le retour à l'inévitable réalité de son mariage forcé aboutissent en revanche à son véritable suicide, révélant ainsi que le mariage d'amour n'est qu'une comédie utopique, un mensonge dissimulant les rapports violents entre les deux sexes.

Dans Oscuro Total, le métathéâtre de Huidobro s'attaque ensuite à la mascarade sociale des relations filiales. De fait, les deux premiers actes de cette pièce présentent deux frères en train d'écrire et de répéter la comédie de la mise à mort de leurs parents. Puis soudainement à l'acte III, les parents surgissent et représentent à leur tour les paroles et les actions de leurs enfants. Ainsi, au fil des jeux spéculaires de Huidobro, les victimes deviennent les bourreaux et seule perdure, à nouveau, la réelle violence, voire le cannibalisme inhérent aux rapports filiaux cubains. «Lo único cierto es la perpetuidad del ritual canibalista dentro del espacio metafórico forjado en base al fingimiento huidizo en la ficción misma, o sea, a la metateatralidad» conclut en effet Jorge Febles (Febles 1997: 113).

Enfin, dans Exilio, Huidobro ébranle même l'espoir de réels liens amicaux en montrant que les retrouvailles finales de cinq amis et hommes de théâtres havanais ne répondent en fait qu'à un plan machiavélique orchestré par l'un d'eux pour jouer, ou peut-être même assassiner réellement, nous ne le saurons jamais, deux autres de ses compères. L'échec de cette diabolique 
comédie en tous cas, ne laisse place, au tomber de rideau, qu'à l'énigmatique image des deux derniers "amis", ou plutôt des deux derniers acteurscomplices, étonnamment tranquilles face aux précédents événements... tout comme, en somme, le paisible vendeur ambulant de Triana qui clôturait déjà le terrible Ceremonial de guerra.

Pourtant, même le sécurisant repli sur soi n'est que vanité et auto-illusion. Le théâtre dans le théâtre cubain parachève en effet son œuvre démystificatrice par la destruction de la fallacieuse image de l'unité de notre propre identité. Il démontre en fait que le Moi de chaque être est aussi un théâtre déchiré par différentes entités antagonistes. Dans Su Cara mitad, en effet, on comprend peu à peu que le violent latino de la pièce interne est moins un être à part entière que l'une des facettes ou des "chères moitiés» de la personnalité de Raúl, l'écrivain immigré. Le Tiznado dont le nom renvoie à l'histoire de l'indépendance cubaine, incarne en fait le Moi hispanique qui habite encore le dramaturge américanisé et qui refusera toujours de le quitter. De même, les autres rôles et «rôles dans le rôle» de Raúl, tels que celui du dramaturge pro-anglais, du latin lover ou de l'hispanique discriminé, ne sont que des Moi stéréotypés qui révèlent aussi la comédie de l'intégration que Raúl se joue à lui-même. Quant aux nombreuses méta-représentations et songes qui rythment Fiesta, et aux multiples emplois qu'y jouent les personnages de Triana, ils ne font que suggérer, eux aussi idéalement, l'émiettement et la fragmentation du Moi de leur metteur en scène, Gerardo. L'auteur le confirme d'ailleurs clairement lorsqu'il dit: «tienen la misma capacidad de darnos lo fragmentario, lo que somos " (in Vasserot 1991: 128).

De la dénonciation de la mise en scène du politique à celle de la collectivité et de l'individualité, le théâtre dans le théâtre cubain détruit donc tout et ne laisse rien sur son passage. Dans la théâtrocratie cubaine, le Moi politique, le Moi social et le Moi profond sont tous intimement liés, car tous traversés par la vaine tentation du coup d'éclat permanent, en référence au titre du célèbre ouvrage de François Mitterrand. L'existence et l'étendue «de ce mal» théâtral ainsi posées, le métathéâtre cubain va pouvoir désormais s'attacher à en montrer les principaux dangers. D'outil de réflexion théorique, les jeux spéculaires insulaires vont alors muter pour devenir très vite des instruments privilégiés de description clinique des symptômes et syndromes psychopathologiques entraînés par l'échec global de cette société du spectacle.

Tout d'abord, le dédoublement sur lequel repose le théâtre dans le théâtre permet aux dramaturges de matérialiser la marginalisation et l'isolement progressif qui guettent les individus dans la comédie politico-sociale 
cubaine. De fait, dès la Noche de los asesinos, Triana situe sa pièce intérieure dans un sótano o un cuarto desván (Triana 1965: 2), c'est-à-dire dans un lieu retiré qui souligne parfaitement le refus de relation des personnages avec leur milieu sociétal. Mieux encore, dans Oscuro total, les comédies intérieures de Huidobro ont lieu dans une résidence à deux étages «en las afueras de una ciudad» où l'on perçoit «a lo lejos, luces distantes de una ciudad» (Montes Huidobro 1997: 117). Ainsi, le jeu de représentations enchâssées, subtilement prolongé par la superposition de deux scènes isolées ainsi que par l'effet de lumières lointaines, matérialise visuellement l'espace opaque de la marge dans lequel se sont aussi plongés ces personnages. Comme le dit René Laforgue, le métathéâtre met donc au premier plan cette zone intermédiaire et asociale, où la culpabilité liée à l'intériorisation de l'échec de la révolution enferme les individus et leur interdit de s'intégrer à nouveau dans le cadre de l'activité collective de leur milieu. Montes Huidobro parfait d'ailleurs somptueusement cette véritable démonstration clinique en abordant dans Las paraguayas le cas le plus extrême de repli du sujet sur soi: l'autisme. De fait, le vieillard de sa pièce perd très vite le contact vital avec la réalité et procède à la reconstruction délirante d'un monde fictif sans aucune communication avec autrui. Confiné dans son délire intérieur de persécution, il ne parvient même plus à entendre la fin des conflits guerriers et ne pense qu'à accomplir jusqu'au bout sa mission première d'extermination en tuant méthodiquement tous ceux qu'il avait épargnés auparavant. Ainsi, comme le dit Jorge Febles, grâce au théâtre dans le théâtre, Huidobro expose «la inevitable culpabilidad del individuo dentro de todo drama colectivo » (Febles 2004: II) et le retrait autistique qu'elle peut entraîner.

Par ailleurs, le métathéâtre met au jour une autre conduite psychotique fondamentale entraînée par l'échec politico-social, celle de l'enfermement dans le passé. En effet, très souvent, on constate que le théâtre dans le théâtre insulaire se présente comme une cérémonie du souvenir qui souligne l'impossibilité d'évolution et de progression des êtres cubains. De fait, dans Exilio, l'homme de théâtre homosexuel qu'est Rubén a beau essayer de s'ancrer dans le présent, il ne fait que revivre et rejouer sans cesse la tragique comédie des persécutions qu'il a subies autrefois à Cuba. Dans son superbe monologue de l'acte II, il dit ainsi à ses amis: «No, esa obra no la van a hacer ustedes [...] porque todavía no han escrito el final. Yo tengo todos los textos escritos sobre mi cuerpo. Letra por letra, sílaba por sílaba, 
palabra por palabra, consigna tras consigna [...] estas heridas que ha escrito la Revolución» (Montes Huidobro 1988: 65) et plus loin il ajoute même:

Todos estos años que ya viví y que los tengo siempre por delante, como si no los hubiera vivido, asfixiándome, estrangulándome [...] porque a mí me han estrangulado desde aquella escena en la cárcel, cuando la Gorda dirigía la obra. Pero ahora voy a terminarla, a dirigirla yo, hasta el final, "good guys" vs “ bad guys” este es mi thriller, Roman! (Montes Huidobro 1988: 82)

Ainsi, grâce à ses jeux métathéâtraux, Huidobro montre que la désillusion et les dérives collectives de la comédie du pouvoir génèrent un puissant symptôme d'ecmnésie chez les Cubains, désormais condamnés, à l'image de Rubén, à revivre comme une expérience toujours actuelle l'échec et les violences passées. Enfin, dans les indications scéniques préliminaires de Fiesta, Triana indique expressément qu'il conçoit sa pièce comme «un juego de la memoria» (Triana 1992: 220). Et bien que la fête théâtrale intérieure reconstitue cette fois un passé cubain à la fois plus ancien et plus heureux, Gerardo finit, ici aussi, par clamer la stérilité de toute cette comédie nostalgique. "Vanidad ¿qué sentido tiene la fiesta? [...] ¡Estoy en un callejón sin salida!»(255). Ainsi, que le métathéâtre plonge les personnages dans un passé traumatique ou idyllique, le constat reste toujours celui de l'immobilisation des êtres due aux troubles mnésiques de l'échec.

Or, toute immobilisation signifie pour l'homme une mort insidieuse. Le panorama métathéâtral des névroses et psychoses engendrées par l'échec politique cubain se termine donc par l'exposition des pulsions masochistes et autodestructrices des individus insulaires. Nombre des structures enchâssées de Montes Huidobro témoignent en effet d'une volonté obstinée des sujets de mettre fin à cette comédie qu'est devenue leur propre vie. Comme Isabel de Funeral en Teruel, comme les personnages d'Oscuro total et les hommes de Las paraguayas, Raúl dans su Cara mitad finit en effet par choisir la mort pour se libérer de son perpétuel sentiment de théâtralité, des Moi qui sont en lui profondément enchâssés et du risque schizophrénique qui y est naturellement lié. Raúl se tue «sólo para evitar responder al [...] Tiznado, su propia voz, su esencia traicionada » confirme en effet Jorge Febles (Febles, in Caballero 2004: 69). Ainsi toute l'œuvre de Huidobro révèle l'impasse mortelle à laquelle conduit le métathéâtre quotidien dans lequel vivent des milliers de Cubains, désireux de composer avec leur réalité désenchantée. Dans ses ouvrages critiques, ce dramaturge en vient même à définir l'assassinat comme une constante fondamentale à tout le corpus métathéâtral insulaire post-révolutionnaire. Citant d'ailleurs Triana, il conclut alors: 
El teatro gradualmente va a ir reflejando esta manifestación patológica de la conducta mediante personajes que proponen la destrucción y que viven en una atmósfera sadomasoquista que es la expresión escénica de la Cuba detrás del telón cuyo montaje es estrictamente castrista. (Montes Huidobro 2008: 121)

Ainsi les rôles dans le rôle et le théâtre dans le théâtre cubain suggèrent bien plus que la comédie du pouvoir insulaire. Ils exposent finalement l'étendue des répercussions ontologiques et des ravages psychologiques de cet échec politique. De la tentation autistique aux troubles mnésiques, des délires schizophréniques aux pulsions sadomasochistes, ils dressent en effet une psychopathologie de l'échec exhaustive et consternante de toute la nation. Cependant, et fort heureusement, une sourde mutinerie menace ce tableau attristant. Partout, en effet, les personnages de ces pièces se relèvent, rebondissent sur ce constat apocalyptique et clament bien haut, à l'image de Gerardo: «En cada hombre existe una chispa de luz, búscala» (Triana 1992: 275). Le dernier volet de notre étude va donc s'attacher à débusquer la paradoxale fertilité de l'échec de la comédie révolutionnaire.

Ce que le métathéâtre cubain révèle surtout, finalement, c'est le merveilleux spectacle de l'espoir résidant dans l'immuable Etat de créativité artistique insulaire et dans les ressources thérapeutiques propres à ses compositions dramaturgiques. De fait, les comédies enchâssées rappellent d'abord sans cesse à cette société qu'elle a su autrefois utiliser la création théâtrale non pas comme un outil de propagande ou de lamentation sociale mais comme un véritable contre-pouvoir propice à la reconstruction personnelle et à la stabilité identitaire des êtres. En multipliant en effet les références à la tradition nationale du teatro bufo et à la force subversive de son choteo, le théâtre dans le théâtre de Triana et Huidobro rappelle clairement au public le caractère salvateur du jeu lorsqu'il est à la fois assumé et permanent face aux événements. Ainsi, dès les premières didascalies de Fiesta, Triana souligne sa volonté de rattacher tous ses spectacles intérieurs à cette contredanse libératrice, à ce cocasse espace de négociation des conflits que fut le théâtre vernaculaire cubain. «El texto, por momentos, debe ser cantado y bailado sin ninguna reserva, tal era lo habitual en el teatro bufo y vernáculo » (220). Plus encore, il achève sa pièce sur un effacement des frontières entre fiction et réalité et sur une provocante rumba qui rappellent encore indubitablement les pouvoirs salvateurs de l'irrespectueux et ambivalent teatro bufo.

Dans Funeral en Teruel, Montes Huidobro multiplie aussi incessamment les références aux danses et aux musiques de ce genre théâtral. Mais surtout, 
c'est le contraste perpétuel entre la spiritualité des pièces internes de Doña Isabel et les expressions populaires des personnages de la pièce externe qui renvoient le plus explicitement aux procédés comiques et démystificateurs de cette tradition déjà métathéâtrale. Escarpenter écrit en effet: "Del teatro bufo cubano, como muy bien apunta el autor al comienzo, la obra toma algunos componentes que responden a "nuestro temperamento nacional distorsionador de la realidad a través de la burla"» (Escarpenter 1990: 12). Ainsi l'espoir que propose finalement ce métathéâtre réside peut-être surtout dans son invitation à réveiller et accepter l'homo ludens qu'a été, qu'est et que sera toujours l'homme cubain. Et Matías Montes Huidobro de conclure: «En cualquier momento nos encontramos al cubano haciendo teatro» (Montes Huidobro 1973: 19).

Plus encore, le métathéâtre cubain prouve dans le détail les vertus thérapeutiques de sa création dramatique en montrant que, paradoxalement, il s'agit bien du seul moyen capable de guérir toutes les conduites psychotiques d'échec, au premier rang desquelles figurait, nous l'avons vu, l'isolement. Les structures enchâssées de Montes Huidobro invitent en effet à s'évader du cadre claustrophobique insulaire et à s'ouvrir à l'altérité en pratiquant des formes d' «échangisme culturel» telles que la transtextualité et la création de nouvelles filiations. De fait, dans Exilio par exemple, ce dramaturge propose un subtil jeu intertextuel non seulement entre l'une de ses pièces internes et La vida es sueño de Caldéron de La Barca mais aussi entre sa pièce externe et Les Bonnes du dramaturge français Genet. De même, dans Funeral en Teruel, le théâtre dans le théâtre d'Isabel est parsemé de citations de Los Amantes de Teruel de Hartzenbusch et surtout d'allusions à Antigone d'Anouilh. Ainsi, la diversité géographique des jeux intertextuels du métathéâtre de Montes Huidobro permet de dépasser les frontières culturelles nationales afin de trouver de nouvelles racines, de nouvelles sources d'inspiration et d'intégrer, in fine, cette nouvelle collectivité qu'est le Tout-Monde. Mieux encore, les créations métathéâtrales cubaines permettent aux personnages et à leur auteur d'accepter les fantômes et cicatrices de l'échec passé afin de s'ouvrir aux perspectives du présent et de l'avenir.

A la fin de Fiesta, en effet, l'échec de la mise en scène d'un passé insulaire harmonieux révèle surtout à cet artiste "en abyme" qu'est Gerardo, qu'il ne pourra se reconstruire qu'en acceptant pleinement ses souvenirs d'exil traumatiques. De fait, l'unité identitaire qu'il recherche aujourd'hui réside précisément dans la dualité de son Moi cubain, marqué à jamais par des âges d'illusion et de désillusion successives, un Moi certes disloqué mais 
assez riche et immuable cependant pour servir, s'il le veut, de fondement au théâtre intérieur de son présent. Et Carolina Caballero de confirmer: «Gerardo will not find the unity and direction he is seeking through a frivolous party or in a re-living of his memories of the past; he realizes that all he seeks is in the present, and inside of him. Despite living in the United States for so many years, he is still very much Cuban» (Caballero 2004: 46). De même, dans Las paraguayas, contrairement à Isabel de Funeral en Teruel, les femmes ne décident pas de s'enfermer dans la comédie d'un amour passé qui les conduirait à se tuer et à nier l'échec et les violences sexuelles qu'elles ont subies. Au contraire, elles choisissent d'en accepter les conséquences et de se reconstruire à partir des enfants qu'elles portent à présent. Évoquant leurs violeurs, La Paraguaya dit en effet:

No los odio, pero han hecho mucho daño. [...] Sólo entendían la vida en la medida de la muerte, el estruendo y la guerra, la conquista y el poder.[...] y sólo fecundaban en la embriaguez de la sangre. Pero yo era la selva. [...] Me tendía sobre la tierra vuelta la tierra misma dando los frutos de mi páramo salvaje y primitivo con la fecundidad de la selva que nos rodea. Nadie podía retenerme porque yo entendía la vida en mi caos de fuego [...]. A veces pienso que somos el barro de Dios (Montes Huidobro 2004: 57-58).

Du lever au tomber de rideau, Huidobro substitue donc aux comédies de la mort les rites de la vie et de la procréation. Il montre ainsi que les femmes peuvent changer les circonstances et le théâtre du monde qui ont fait d'elles des victimes, pour reconstruire un nouveau système et une nouvelle scène fondés sur des valeurs plus nobles, plus humanistes, et traditionnellement plus féminines. Jorge Febles ne dit-il pas, en effet, à ce sujet: "Sólo la transformación del hombre como tal, su amable feminización a la sombra de la mujer, promovería la luminosa purificación progresista» (Febles Jorge 2004: IX).

Qu'on ne croie pas pour autant que l'auteur cherche ici à délivrer et inculquer une nouvelle vérité féministe ou révolutionnaire. Car enfin, et ce sera notre dernier point, si le métathéâtre et la création dramaturgique cubaine sont porteurs d'espoir, c'est bien parce qu'ils permettent de faire comprendre au peuple cubain que la vérité, en tant que telle, n'existe pas, que ce qui existe à l'infini, que ce qui pourrait éventuellement s'appeler vérité, c'est la manière que chacun - et a fortiori chaque auteur -, a de décrire et de penser une réalité donnée. La fiction, la réalité, la vérité, le mensonge: tout cela, en fait, n'a plus le moindre sens pour nos dramaturges cubains car ce qui les intéresse justement dans le métathéâtre, c'est l'incroyable capacité de cette structure à exprimer le plus fidèlement ce qu'ils pensent 
et ce qu'ils voient tout en exhibant fièrement la part essentiellement relative et subjective de leur jugement. Bref, ce qui les fascine et ce qui nous fascine aussi, plus que tout, dans cette technique spéculaire, c'est l'espoir qu'elle révèle résidant dans la sincérité de la création dramaturgique et artistique.

Montes Huidobro et Triana savent en effet qu'ils peuvent se tromper sur la réalité (qu'ils prennent pour de la fiction, et vice-versa), ils savent qu'ils peuvent se méprendre sur ce qu'ils pensent être définitivement la vérité castriste et l'ère post-révolutionnaire, mais ce que leur métathéâtre révèle, c'est qu'ils ne peuvent tricher, ils ne peuvent s'arranger avec la sincérité créatrice. Et tant mieux, car au présent, face au permanent mensonge politique castriste, ce que les hommes de théâtre cubains peuvent offrir de mieux, et savent offrir de mieux, au-delà de leur fiction réelle, de leur réalité fictive, de leur vérité mensongère, de leur mentir-vrai, c'est bien leur sincérité. Voilà pourquoi Triana finit par faire clamer à Aracelio dans Ceremonial de guerra qu'il n'existe que sa propre «vérité » (Triana 1990: 57). Voilà pourquoi Montes Huidobro dira aussi: «uno tiene que vivir con lo que uno ha elegido, que es el único modo de ser. $\mathrm{Y}$ en última instancia, como en el teatro, es la única verdad posible» (Montes Huidobro 1997: 44). À notre tour donc de proclamer que l'espoir réside dans cette mince mais pure sincérité révélée par le métathéâtre des créations dramaturgiques cubaines. À notre tour, in fine, d'annoncer bien haut au peuple cubain qu'il existe encore dans l'art une forme de pureté qui pourra peut-être l'aider à se relever.

"L'échec, toujours essentiel, nous dévoile à nous-mêmes» disait Cioran. J'espère donc que cette brève étude sur le métathéâtre cubain est un échec car, si elle en est un, elle aura réussi à révéler le caractère vital de cette technique dramaturgique pour les auteurs et pour le peuple insulaire. Elle aura montré que des spectacles métathéâtraux de l'échec de la théâtrocratie cubaine, surgit, de façon inespérée, une incroyable fertilité; oui, le théâtre dans le théâtre selon Triana et Huidobro propose un véritable espoir, oui, il ouvre à une possible salvation, parce qu'il montre finalement qu'il n'existe pas plus de vérité que d'échec absolu, parce qu'il invite ainsi implicitement son public à se redresser en élaborant, à son tour, avec une exemplaire sincérité, sa propre vérité artistique du monde. 


\section{Bibliographie:}

Caballero, Carolina, 2004, The Performance of Exile in Selected Plays by Matías Montes Huidobro, José Triana, Pedro Monge Rafuls, and Alina Troyano, thèse soutenue à l'Université de North Carolina, Romance Languages, Spanish, (sous la direction de María A. Salgado et Stuart A. Day), p. 46-96.

Escarpenter, José, 1990, "Funeral en Teruel y el concepto de la hispanidad», in Funeral en Teruel, Honolulu, Hawaii, Persona, "Serie teatro», p. 11-14.

Febles, Jorge, 1997, "Re-textualización y rito en Oscuro total», in Ollantay theater magazine, v. 2, Summer/Autumn 1997, p. 106-114.

Febles, Jorge, 2004, «Las paraguayas en su contexto », in Las paraguayas, Milwaukee, Caribe, p. I-XI.

Montes Huidobro, Matías, 1973, Persona, vida y máscara en el teatro cubano, Miami, Florida, Universal.

Montes Huidobro, Matías, 1988, Exilio, Honolulu, Hawaii, Persona, «Serie Teatro».

Montes Huidobro, Matías, 1990, Funeral en Teruel, Honolulu, Hawaii, Persona, «Serie Teatro».

Montes Huidobro, Matías, 1992, "Su Cara mitad », in Carlos Espinosa Domínguez, Teatro cubano contemporáneo, Antología, Madrid, Fondo de cultura contemporáneo, p. 632-703.

Montes Huidobro, Matías, 1997, «Oscuro Total», in Ollantay Theater Magazine, V.2, Summer/Autumn, p. 117-195.

Montes Huidobro, Matías, 1997, "Escribir teatro en el exilio", in Ollantay Theater Magazine, V 5.2.

Montes Huidobro, Matías, 2004, Las paraguayas, Milwaukee, Caribe.

Montes Huidobro, Matías, 2008, Cuba detrás del telón II, el teatro entre la estética y el compromiso (1962-1969), Miami, Florida, Universal.

Triana, José, 1965, La noche de los asesinos, La Habana, Casa de las Américas.

Triana, José, 1990, Ceremonial de guerra, Honolulu, Hawaii, Persona, «Serie Teatro».

Triana, José, 1992, Fiesta, in Teatro: 5 autores cubanos, New York, Ollantay, p. 217-277.

Vasserot, Christilla, 1991, "Entretien avec José Triana», in Latin American Theatre Review, University of Kansas, Center of Latin American Studies, Autumn 1995, $\mathrm{n}^{\circ} 291$. 